\title{
2D Axisymmetric Modelling of Single Liquid Droplet Impingement at High Speeds on Thin Liquid Films in Compressible Regime
}

\author{
Mason Marzbali ${ }^{1,2}$, Ali Dolatabadi ${ }^{2}$ \\ ${ }^{1}$ Department of Mechanical Engineering, American University in Dubai \\ P.O. Box 28282, Dubai - United Arab Emirates \\ mmarzbali@aud.edu; ali.dolatabadi@concordia.ca \\ ${ }^{2}$ Department of Mechanical, Industrial and Aerospace Engineering, Concordia University \\ 1455 De Maisonneuve Blvd. W., Montreal, QC, H3G 1M8, Canada
}

\begin{abstract}
High-speed droplet impact is of great interest to power generation and aerospace industries due to the accrued cost of maintenance in steam and gas turbines. The repetitive impacts of liquid droplets onto rotor blades, at high relative velocities, result in the blade erosion, which is known as Liquid Impingement Erosion (LIE). Experimental and analytical studies in this field are limited due to the complexity of the droplet impact at such conditions. Hence, numerical analysis is a very powerful and affordable tool to investigate LIE phenomenon. In this regard, it is crucial to understand the hydrodynamics of the impact in order to identify the consequent solid response before addressing the LIE problem. The numerical study of the droplet impingement provides the transient pressure history generated in the liquid. Knowing the transient behavior of the substrate, in response to pressure force exerted due to the impact, would facilitate engineering new types of surface coatings that are more resistant to LIE. In LIE problem, a scenario may occur where the previously impinged droplet has spread over the surface and the subsequent impinged droplet impacts a thin liquid film that was formed on the substrate. For this reason, the droplet impingement is simulated with compressible VOF solver for the impact of a $500-\mu \mathrm{m}$ droplet impinged at $350 \mathrm{~m} / \mathrm{s}$ onto a liquid film with a thickness of $100 \mu \mathrm{m}$ and the results obtained from the compressible fluid modeling are compared with the case of a dry substrate under the same impact condition.
\end{abstract}

Keywords: Liquid impingement erosion, compressible droplet impact, impact on liquid film, CFD, VOF

\section{Introduction}

Although the experimental study of droplet impacts dates back to 1876 by the work of Worthington [1], it still remains an area of extensive study today. The advancement of high-speed imaging techniques, in addition to computational power, have allowed researchers to obtain much higher temporal and spatial resolutions in capturing the hydrodynamics of droplet impingement. There are various physical phenomena that occur upon the impact of a liquid droplet on a solid substrate, such as spreading, fingering, air entrapment, bouncing and splashing, that drew the attention of various industries and academia. For instance, the spreading of a droplet at low impingement velocities has been studied in details by Fukai et al. [2], Chandra and Avedisian [3], Pasandideh-Fard et al. [4] and more recently by Roisman et al. [5]. The entrapment of an air bubble underneath the droplet has been simulated by Mehdi-Nejad et al. [6]. Moreover, the formation of fingers upon impact of water drops was experimentally investigated by Mehdizadeh et al. [7]. Blake and De Conick [8] have extended the molecular-kinetic theory of dynamic wetting by considering the effect of fluid-solid interaction. A 2-D axisymmetric, two-way coupled Fluid-Solid Interaction model for impingement of liquid droplets onto rigid and elastic Ti-6Al-4V substrate was presented by Marzbali [9], Marzbali et al. [10] and Marzbali \& Dolatabadi [11]. It was illustrated that the fluid compressibility effect on the pressure build-up and solid stress cannot be neglected for impingement velocities of 100 $\mathrm{m} / \mathrm{s}$ and above. However, most of the past studies in droplet impingement are concerned with low impact velocities, hence, do not take into account the fluid compressibility, which becomes critical at high impingement velocities.

Upon the impact of a liquid droplet on a solid substrate at a high velocity, compression waves begin to propagate in the droplet due to the fluid compressibility. These compression waves create a high density region in the liquid close to the solid surface and break away from the droplet periphery, giving rise to the formation of lateral jets, as reported by Heymann [12] and confirmed by the experiments of Dear et al. [13] and Field et al. [14]. The pressure waves generated in the liquid accumulate over time and form a high-pressure front since the speed of sound changes in the compressed region. 
Compression waves travel through the compressed region at the speed of sound in the liquid, hence, the rest of the droplet, outside the disturbed region, is not affected by the impact yet. The pressure and density inside the undisturbed region remain unchanged since it is completely unaware of the impact. As the liquid droplet continues its downward motion toward the solid, the high-pressure front penetrates further into the liquid bulk and the compressed region increases in size. At the same time, the droplet contact line is moving radially outward from the droplet center axis, right after the droplet touches down on the surface. Initially, the contact periphery spreads out much faster than the high-pressure front. As the contact line starts to decelerate due to viscous dissipation, it is finally overtaken by the high-pressure front that is spreading outward. At this exact instant, the high-pressure front detaches and starts to travel along the free surface leading to the formation of lateral jets. This jetting eruption is the result of a very large difference between the pressure and density across the free surface of the droplet in contact line region. When lateral jetting occurs, the impact pressure reaches its temporal maximum value, as experimentally measured for the first time by Rochester and Brunton [15]. It has been shown by Heymann [12] that the pressure in the compressed area is not uniform and the location where the highest pressure appears is behind the contact line.

Lesser and Field [16] studied the droplet impact analytically and in order to determine the exact position of the high-pressure front, an envelope of individual wavelets emitted by the expanding contact edge was proposed. In their model, it was assumed that the compression wave velocity is equal to the speed of sound in ambient condition. Although this assumption is valid for the acoustic limit, Haller [17] demonstrated that it is invalid for high impact velocities where the liquid compressibility effect is considerable. He has shown, both computationally and analytically, that the compression wave velocity during the first stage of the impact, at an impingement velocity of 500 $\mathrm{m} / \mathrm{s}$, is in the range of 2600-3000 m/s, which is significantly higher than the ambient speed of sound in water, i.e., $1430 \mathrm{~m} / \mathrm{s}$. Therefore, in modeling the compressible droplet impact, it is essential to account for the changes in the speed of sound, via the variation of liquid density, with an equation of state. In this regard, a compressible Volume of Fluid (VOF) model was utilized to resolve the interfacial gas-liquid flow for droplet impingement on dry rigid substrate and the results were published by Marzbali \& Dolatabadi [18]. The obtained results for lateral jet velocity, liquid density and maximum pressure were compared with the study reported by Haller [17] previously. The main objective of the current work is to study the effect of liquid film thickness on the pressure build-up via high-speed droplet impingements. To that end, the impact condition that occurs frequently in liquid impingement erosion problem, i.e., droplet diameter of $500 \mu \mathrm{m}$ and impingement velocity of $350 \mathrm{~m} / \mathrm{s}$ is selected. The liquid film thickness is $100 \mu \mathrm{m}$, which results in a film thickness to droplet diameter ratio of 0.2. Finally, the maximum pressure exerted on the solid surface due to the droplet impact is calculated for both dry and wetted substrates and the results are compared with each other.

\section{Methodology}

\subsection{Governing equations}

Navier-Stokes equations are solved for two compressible and immiscible fluids. The conservation of mass and momentum equations in their transient and compressible forms are as follows,

$$
\begin{gathered}
\frac{\partial \rho_{f}}{\partial t}+\nabla \cdot\left(\rho_{f} V_{f}\right)=0 \\
\frac{D\left(\rho_{f} V_{f}\right)}{D t}=\nabla . \sigma_{f}+\rho_{f} F_{b}
\end{gathered}
$$

where $V_{f}$ is the fluid velocity vector, $\rho_{f}$ is the fluid density, $F_{b}$ is body forces acting on the fluid. $\sigma_{f}$ is the Cauchy stress tensor for a Newtonian fluid, including pressure and viscous terms, defined as,

$$
\sigma_{f}=-p_{f} I+\tau_{f}
$$

where $p_{f}$ is the fluid pressure, $I$ is the 3 by 3 identity matrix and $\tau_{f}$ is the viscous stress tensor,

$$
\tau_{f}=\mu_{f}\left(\nabla V_{f}+\nabla V_{f}^{T}\right)-\frac{2}{3} \mu_{f}\left(\nabla \cdot V_{f}\right) I
$$


where $\mu_{f}$ is the fluid dynamic viscosity. A single momentum equation is solved for both gas and liquid phases and fluid properties are calculated for the mixture. The fluid flow is assumed to be laminar; hence, no turbulence model is employed in the solver. The energy equation for a compressible flow is as follows,

$$
\frac{D\left(\rho_{f}\left[e+V_{f}^{2} / 2\right]\right)}{D t}+\nabla \cdot\left(V_{f} p_{f}\right)=-\nabla \cdot q+\nabla \cdot\left(\tau_{f}\right) \cdot V_{f}+\rho_{f} g \cdot V_{f}
$$

where $e=c T$ is the internal energy and $q$ is the heat flux vector, calculated based on the gradient of the internal energy,

$$
q=-\alpha \nabla e
$$

where $\alpha$ is the thermal diffusivity. In addition, in order to close the system of equations, an equation of state for gas and liquid phases is needed. The ideal gas law is applied to account for the air compressibility as follows,

$$
p_{g}=\rho_{g} R T_{g}
$$

where $R$ is the specific gas constant equal to $287 \mathrm{~J} / \mathrm{kgK}$ for air. For the liquid phase, the power law equation of state proposed by Tait [19], commonly used for water, is utilized and defined below,

$$
\frac{p_{l}+B}{p_{a}+B}=\left(\frac{\rho_{l}}{\rho_{l_{0}}}\right)^{N}
$$

where $\rho_{l_{0}}$ is the density of water at ambient conditions equal to $1000 \mathrm{~kg} / \mathrm{m}^{3}$ and $B, p_{a}$ and $N$ are three constants from Tait's equation [19] equal to $B=300 M P a, p_{a}=0.1 M P a$ and $N=7.415$, respectively.

The surface tension force is treated as a pressure gradient across the liquid-gas interface and is calculated per unit volume based on the Continuum Surface Force (CSF) model proposed by Brackbill et al. [20]. The compressible VOF model adapted from Hirt and Nichols [21] is employed to resolve the droplet interface. In VOF method, a scalar field is defined for volume fraction of the liquid phase, namely $\alpha$, and its value depends on the fraction of the cell volume occupied by this phase. The values between zero and one denote the interface between gas and liquid phases. Since the volume fraction represents the volume occupied by the liquid, it should be advected by the flow field at each time step. The following equation governs the liquid advection,

$$
\frac{\partial\left(\rho_{f} \alpha\right)}{\partial t}+\nabla \cdot\left(\rho_{f} \alpha V_{f}\right)=0
$$

Following the advection, the interface is reconstructed using the Piecewise Linear Interface Calculation (PLIC) proposed by Youngs [22]. In PLIC method, the interface is defined at each computational cell by a slope and an intercept. The slope of the interface is calculated based on the volume fractions of the neighboring cells.

\subsection{Computational domain, boundary and initial conditions}

The computational domain for the 2-D axisymmetric model has a width of eight times the droplet radius $(R)$ and the height of the domain is $4 R$. The gravitational force is exerted in the same direction as the droplet impingement. The computational domain consists of air and water phases and the properties at ambient conditions are utilized for both phases. Outflow boundary condition is applied to all fluid boundaries except for the substrate surface. No-slip condition is imposed on the fluid-solid interface. In addition, the droplet center axis has axisymmetric boundary condition.

Initially, the fluid domain is filled with liquid film at the bottom and air elsewhere. At the beginning of each simulation the droplet is patched in the fluid domain with the desired size and velocity. The computation for the rest of the cells starts with zero for the velocity and liquid volume fraction in the air. The initial temperature and pressure in all the computational cells including the boundaries are set to the ambient conditions, i.e. $300 \mathrm{~K}$ and $100 \mathrm{kPa}$, respectively. 


\subsection{Numerical schemes and discretization}

The Pressure-Implicit with Splitting of Operators (PISO) method is used for pressure-velocity coupling in transient calculations. The integral forms of fluid equations are discretized over each control volume and solved for a fixed system of grids in a segregated manner. The solution is then obtained by using a time marching scheme based on the given initial condition. The Gaussian integration method is used for summation of the values on cell faces interpolated linearly from cell centers. The time scheme is first-order implicit based on Euler and is bounded. The gradient schemes are Gauss linear with central differencing. The divergence schemes are also Gaussian with an upwind discretization for all the transport equations except for volume fraction flux which is second-order accurate in space and unbounded. Finally, the uncorrected Gauss linear scheme is utilized in discretizing the Laplacian equation. For other variables, such as temperature, density and kinetic energy, an upwind method, which is first order and bounded, is used to discretize the equations. The second derivative of temperature is discretized with a linear scheme, which is second order and unbounded. The linear Gaussian scheme is utilized for interpolation from the cell centers to the faces.

The time step during for all the advective fluxes in transport equations is different from the one used in VOF calculations. The time step in VOF is adaptive and depends on the Courant-Friedrichs-Lewy (CFL) condition. The maximum CFL is set to 0.1 during the computations to constrain the adaptive time step. This requirement ensures that the liquid advection through one cell is less than $10 \%$ at every time step and the initial time step is determined based on the impact velocity and grid size, depending on the impact condition. Finally, OpenFoam, an open-source computational code, is used for programming the solver. In OpenFoam, all the equations are solved in three dimensions and a 2-D axisymmetric mesh is simply a $5^{\circ}$ section of a full cylinder with flat sides and one cell in lateral direction, which results in a wedge. In presenting certain results, the pressure is nondimensionalized as $p^{*}=p / p_{w h}$, where $p_{w h}=\rho_{0} V_{0} c$ is the water hammer pressure, $\rho_{0}=1000 \mathrm{~kg} / \mathrm{m}^{3}$ is density of undisturbed water, $V_{0}$ is impact velocity and $c$ is the speed of sound in undisturbed water which is equal to $1430 \mathrm{~m} / \mathrm{s}$.

\section{Results and discussion}

The impact of a droplet with a dimeter of $500 \mu \mathrm{m}$ and an impingement velocity of $350 \mathrm{~m} / \mathrm{s}$ onto a liquid film with a thickness of $h=100 \mu \mathrm{m}(h / D=0.2)$ is considered first. The contours of pressure (left) and velocity (right) are presented in Fig. 1 over eight time steps. In addition, the contours of density (left) and temperature (right) are displayed in Fig. 2. As it can be seen in Fig. 1, a high-pressure region is formed at $t=3$ ns within the droplet and almost mirrored in the liquid film, however, its envelope is larger inside the film. Figure 2 reveals that the density rises in the compressed region and the highest density appears in a narrow region (in red) between the droplet edge and liquid film. The temperature in this area also increases. At $t=5 \mathrm{~ns}$, the high-pressure front penetrates further down the liquid film, shown in Fig. 1, and the highest velocity and pressure are observed around the droplet edge. The pressure front reaches the solid surface by $t=7 \mathrm{~ns}$ and a secondary compressed region is formed in the liquid, illustrated in figures 1 and 2. However, the maximum density is still located at the droplet-film interface.

The gas-liquid interface, presented in Fig. 3, demonstrate that the high-density dots, observed in Fig. 2, are in fact air bubbles that have been trapped between the droplet interface and liquid film upon the impact. The compressed region inside the liquid film keeps growing until $t=11 \mathrm{~ns}$, when it reaches the initial liquid film surface. At the same moment, the lateral jetting commencement can be observed in figures 1 and 2 . The temperature also rises in the jet and the liquid attached to it. At $t=13 \mathrm{~ns}$, the compression waves that reached the liquid free surface are projected back in the opposite direction inside the liquid as expansion waves. The interaction between the compression and expansion waves results in stripes of high and low pressures that can be clearly seen in Fig. 1. Figures 1 and 2 demonstrate that the lateral jet erupted from the droplet periphery in contact with the liquid film is associated with a high velocity and temperature because of the momentum and heat transfer in that region. Figure 3 clearly shows that the eruption of the lateral jet from the edges has already begun and the formation of smaller droplets due to the jet breakup is evident. It is worth mentioning that the formation of the lateral jet is delayed upon the impact on a liquid film as opposed to a rigid substrate. This is due to the fact that the liquid film is easily deformed by the impinging droplet and the kinetic energy is transferred from the droplet to the liquid film. As a result, the stagnation region is not fully developed until the pressure front reaches the solid surface, i.e., at $t=5 \mathrm{~ns}$. After this time, the radial flow is enhanced as the stagnation region grows in the liquid and the eruption of the lateral jet can be observed at $t=11 \mathrm{~ns}$. 

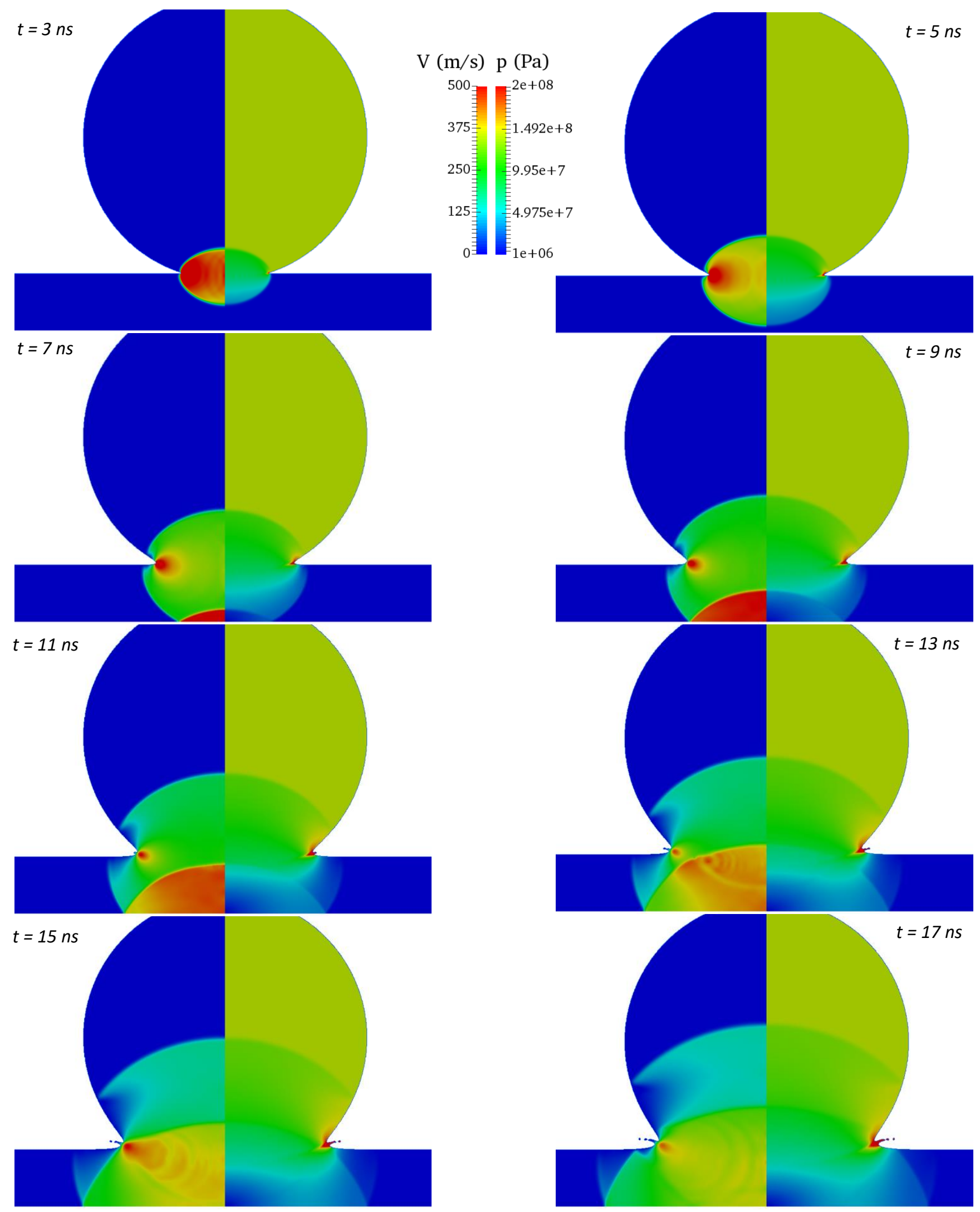

Fig. 1: Contours of pressure (left) and velocity (right), $D=500 \mu \mathrm{m}, V_{0}=350 \mathrm{~m} / \mathrm{s}, h / D=0.2$. 


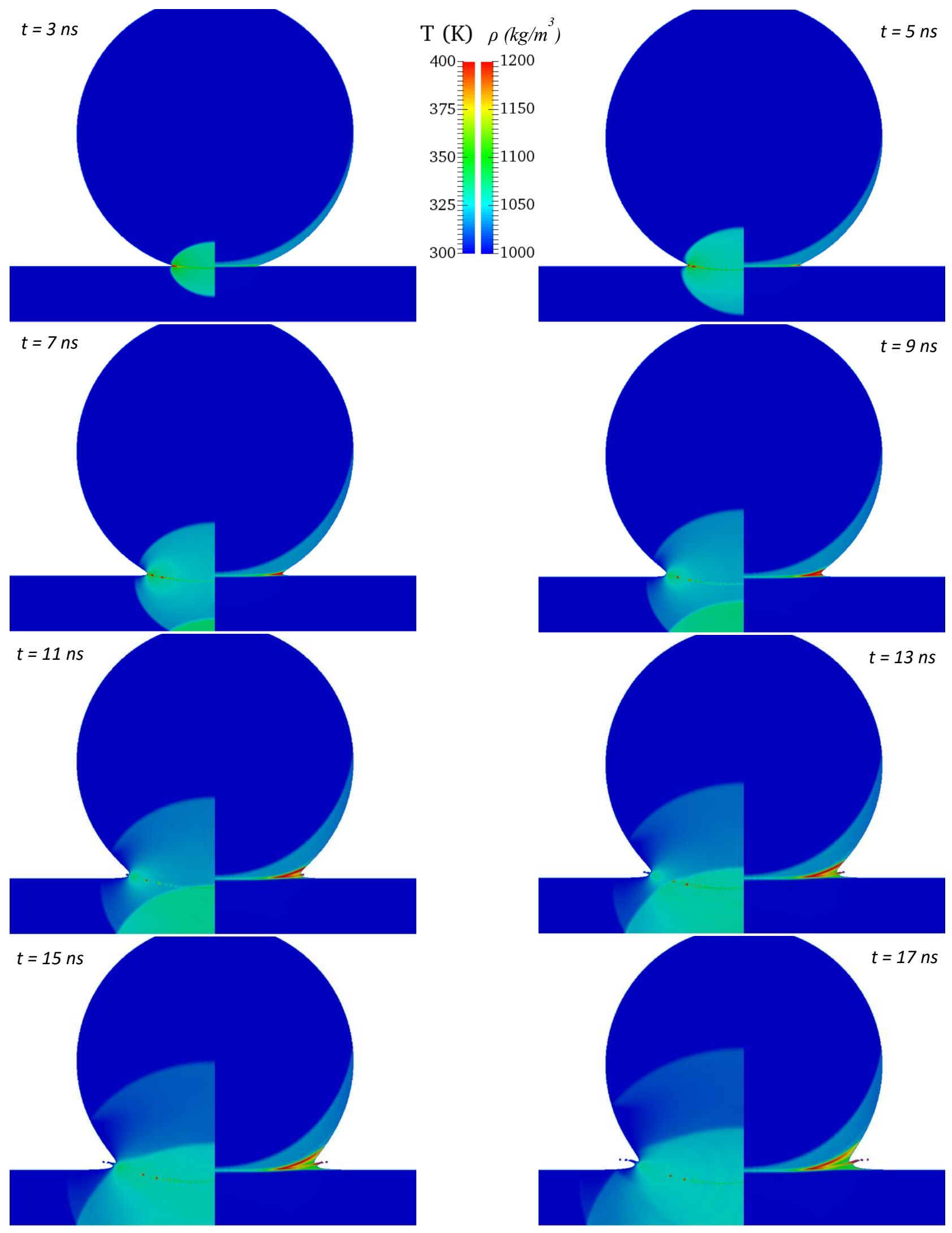

Fig. 2: Contours of density (left) and temperature (right), $D=500 \mu \mathrm{m}, V_{0}=350 \mathrm{~m} / \mathrm{s}, h / D=0.2$. 

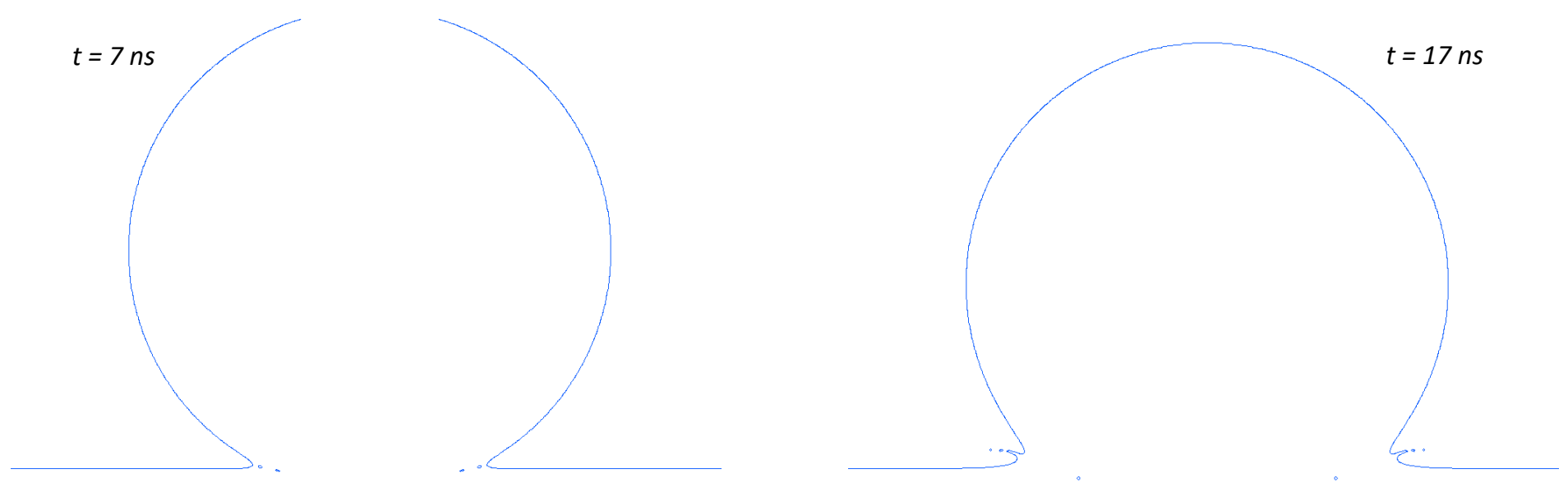

Fig. 3: Gas-liquid free surface at $t=7$ and $17 \mathrm{~ns}, D=500 \mu \mathrm{m}, V_{0}=350 \mathrm{~m} / \mathrm{s}, h / D=0.2$.

The maximum pressure exerted on the substrate after the droplet impact on 100- $\mu$ m liquid film is $225 \mathrm{MPa}\left(p^{*}=0.45\right)$ which is 5 times less than the pressure build-up upon impingement of the same droplet on a dry substrate, which is equal to $1125 \mathrm{MPa}\left(p^{*}=2.25\right)$, as reported previously [18]. As a parametric study, 11 film thickness to droplet diameter ratios, ranging from 0.02 up to 0.4 , corresponding to a film thickness of 10 to $200 \mu \mathrm{m}$, with the same droplet size of $500 \mu \mathrm{m}$ and impingement velocity of $350 \mathrm{~m} / \mathrm{s}$ were modeled. The maximum pressure imposed on the surface diminishes significantly with respect to the film thickness. For instance, the maximum pressure is equal to the water hammer pressure (500 MPa) for $h / D=0.04(h=20 \mu \mathrm{m})$ and half of the water hammer pressure $(250 \mathrm{MPa})$ for $h / D=0.16(h=80 \mu \mathrm{m})$. In other words, the liquid film partially absorbs the impact force and reduces the pressure build-up on the substrate, thus the subsequent stress under the solid surface would decrease substantially. A correlation based on a logarithmic function of film thickness and a linear dependence on the impact Mach number can be obtained by simulating a wide range of impingement scenarios with various impact velocities, droplet diameters and liquid film thicknesses. These results will be published in near future.

\section{Conclusion}

In summary, the droplet impact onto a 100- $\mu$ m liquid film was modeled utilizing compressible VOF solver for an impact velocity of $350 \mathrm{~m} / \mathrm{s}$ and a droplet size of $500 \mu \mathrm{m}$. The results revealed that when a liquid droplet is impinged on a liquid film, low pressure bands are formed inside the liquid due to reflection of the compression waves on the liquid surface as expansion waves and their cancellation effect. Furthermore, the formation of the lateral jet is delayed due to the damping effect and the stagnation region is not developed until the pressure front reaches the solid surface. Air bubbles that are compressed due to the high impact pressure are trapped between the droplet interface and liquid film upon the impact.

The maximum generated pressure on the solid is reduced significantly with increasing the film thickness, as the liquid film partially absorbs the impact force. It was shown that the impact pressure is reduced five times if the droplet is impinged onto a $100-\mu \mathrm{m}$ liquid film as opposed to a dry substrate. Moreover, it is likely that cavitation happens at the droplet periphery due to the high temperature $(\sim 400 \mathrm{~K})$. As a continuation of the present work, the phase change model can be added to the solver to study the cavitation phenomenon in more detail.

\section{Acknowledgements}

This work was sponsored by Concordia University, Consortium de Recherche et d'Innovation en Aérospatiale au Québec (CRIAQ), Natural Sciences and Engineering Research Council of Canada (NSERC), Nano-Quebec, Hydro Quebec and Siemens Canada. Computations were made on the supercomputer "Mammouth-Mp2b" from "uSherbrooke", managed by Calcul Québec and Compute Canada. The operation of this supercomputer is funded by the Canada Foundation for Innovation (CFI), the ministère de l'Économie, de la science et de l'innovation du Québec (MESI) and the Fonds de recherche du Québec - Nature et technologies (FRQ-NT). 


\section{Reference}

[1] A. Worthington, "On the forms assumed by drops of liquids falling vertically on a horizontal plate," in Proceedings of the Royal Society of London, 1876.

[2] J. Fukai, Y. Shiiba, T. Yamamoto, O. Miyatake and D. Pouli, "Wetting effects on the spreading of a liquid droplet colliding with a flat surface: experiment and modeling," Physics of Fluids, vol. 7, no. 2, 1995.

[3] Chandra S. and C. T. Avedisian, "On the Collision of a Droplet with a Solid Surface," Proceedigs of the Royal Society, vol. 432, no. 1884, 1991.

[4] M. Pasandideh-Fard, . Y. M. Qiao, S. Chandra and J. Mostaghimi, "Capillary effects during droplet impact on a solid surface," Physics of Fluids, vol. 8, no. 3, 1996.

[5] I. V. Roisman, R. Rioboo and C. Tropea, "Normal impact of a liquid drop on a dry surface: model for spreading and receding," Proceedings of the Royal Society, vol. 458, no. 2022, 2002.

[6] V. Mehdi-Nejad, J. Mostaghimi and S. Chandra, "Air bubble entrapment under an impacting droplet," Physics of fluids, vol. 15, no. 1, pp. 173-183, 2003.

[7] N. Z. Mehdizadeh, S. Chandra and J. Mostaghimi, "Formation of fingers around the edges of a drop hitting a metal plate with high velocity," Journal of Fluid Mechanics, vol. 510, pp. 353-373, 2004.

[8] T. D. Blake and J. De Conick, "The influence of solid-liquid interactions on dynamic wetting," Advances in Colloid and Interface Science, vol. 96, no. 1-3, pp. 21-36, 2002.

[9] M. Marzbali, "Numerical Analysis of High-speed Droplet Impingement on Elastic and Rigid Substrates," Concordia University, 2017.

[10] M. Marzbali, A. Dolatabadi and P. Jedrzejowski, "Fluid-Solid Interaction Modeling of Compressible Droplet Impact onto Elastic Substrates," in 21st AIAA Computational Fluid Dynamics Conference, San Diego, 2013.

[11] M. Marzbali and A. Dolatabadi, "Modeling of Liquid Droplet Impingement onto Ti-6Al-4V Substrate," in The 5th World Congress on Mechanical, Chemical, and Material Engineering, Lisbon, 2019.

[12] F. J. Heymann, "High-speed impact between a liquid drop and a solid surface," Journal of Applied Physics, vol. 40, pp. 5113-22, 1969.

[13] J. P. Dear and J. E. Field, "High-speed photography of surface geometry effects in liquid/solid impact," Journal of Applied Physics, vol. 63, pp. 1015-21, 1988.

[14] J. E. Field, J. P. Dear and J. E. Ogren, "The Effects of Target Compliance on Liquid Drop Impact," Journal of Applied Physics, vol. 65, pp. 533-40, 1989.

[15] M. C. Rochester and J. H. Brunton, "Erosion by Liquid and Solid Impact," in International Conference on Erosion by Liquid and Solid Impact (5th), Cambridge, 1979.

[16] M. B. Lesser and J. E. Field, "The Impact of Compressible Liquids," Annual Review of Fluid Mechanics, vol. 15, pp. 97-122, 1983.

[17] K. Haller, "High-Velocity Impact of a Liquid Droplet on a Rigid Surface: The Effect of Liquid Compressibility," Swiss Federal Institure of Technology, Zurich, 1972.

[18] M. Marzbali and A. Dolatabadi, "High-speed Droplet Impingement in Compressible Regime," in Proceedings of the 5th World Congress on Mechanical, Chemical, and Material Engineering, Lisbon, 2019.

[19] P. G. Tait, "Report on some of the physical properties of fresh water and sea water," Physical Chemistry, vol. 2, p. $1-17,1888$.

[20] J. U. Brackbill, D. B. Kothe and C. Zemach, “A continuum method for modelling surface tension,” vol. 100, no. 2, p. $335-354,1992$.

[21] C. Hirt and B. Nichols , "Volume of Fluid (VOF) Method for the Dynamics of Free Boundaries," vol. 39, no. 1, pp. 201-225, 1981.

[22] D. L. Youngs, "Time-Dependent Multi-Material Flow with Large Fluid Distortion,” Numerical Methods for Fluid Dynamics, 1982. 